\title{
Hydroxysafflor Yellow A inhibits the viability and migration of vascular smooth muscle cells induced by serum from rats with chronic renal failure via inactivation of the PI3K/Akt signaling pathway
}

\author{
CHANGLIANG HUO*, LI WANG*, QIULI WANG*, YANBO YANG and BO CHEN \\ Department of Nephrology, Lianyungang Hospital of Traditional Chinese Medicine, Lianyungang, Jiangsu 222000, P.R. China
}

Received October 30, 2020; Accepted May 19, 2021

DOI: $10.3892 /$ etm.2021.10282

\begin{abstract}
It has been reported that the viability and migration of vascular smooth muscle cells contributes to arteriovenous fistula stenosis. Hydroxysafflor Yellow A (HSYA) has been demonstrated to inhibit the viability and migration of VSMCs by regulating Akt signaling. The present study aimed to investigate the role of HSYA on the viability and migration of human umbilical vein smooth muscle cells (HUVSMCs) following stimulation using serum from rats with chronic renal failure (CRF), and to determine the effects of HSYA on PI3K/Akt signaling. Wistar rats were randomly divided into two groups, control and CRF groups. Serum from each group was collected to stimulate the HUVSMCs. Cell Counting Kit-8 and wound healing assays were performed to assess cell viability and migration, respectively. Flow cytometry analysis was performed to assess apoptosis, and western blot analysis was performed to detect protein expression levels of PI3K and Akt. Nitric oxide (NO) production was measured using the Nitrate/Nitrite assay kit. The results demonstrated that serum from CRF rats significantly enhanced cell viability, migration and apoptosis, the effects of which were reversed following treatment with HSYA. In addition, CRF serum decreased NO and endothelial NO synthase expression, whilst increasing the protein expression levels of PI3K and phosphorylated-Akt in HUVSMCs. Notably, treatment with HSYA markedly restored NO production and inactivated the PI3K/Akt signaling pathway. Furthermore, the PI3K/Akt inhibitor, AMG511, exerted similar effects to HSYA. Taken together, the results of the present study suggest that HSYA suppresses cell viability
\end{abstract}

Correspondence to: Dr Bo Chen, Department of Nephrology, Lianyungang Hospital of Traditional Chinese Medicine, 160 Chaoyang Middle Road, Haizhou, Lianyungang, Jiangsu 222000, P.R. China

E-mail: drchenbo@yeah.net

*Contributed equally

Key words: Hydroxysafflor Yellow A, arteriovenous fistula, vascular smooth muscle cells, migration, viability and migration in the presence of CRF serum by inactivating the PI3K/Akt signaling pathway.

\section{Introduction}

Hemodialysis is the primary renal replacement therapy for patients with end-stage renal disease (ESRD), and is designed to perform some of the functions lost as a result of chronic renal failure (CRF), such as clearing metabolic wastes and regulating the balance of water, electrolytes and the acid-base balance, which increases the survival of patients and improves the quality of life (1). Arteriovenous fistula (AVF) is the preferred mode of access for hemodialysis. The artery near the wrist of the forearm and the adjacent vein are sutured to allow arterial blood flow in the vein following anastomosis, which forms the AVF (2), the 'lifeline' for patients with hemodialysis. However, the patency rate of AVF after 1 year is $70 \%$, and $48 \%$ after 4 years (3). The failure of AVF can be attributed to venous stenosis, intimal hyperplasia, technical problems and inflow issues (4-6).

The primary cause of AVF stenosis is venous stenosis caused by intimal hyperplasia at the anastomosis site of AVF (7). Previous studies have reported that intimal hyperplasia is predominantly caused by the viability and migration of vascular smooth muscle cells (VSMCs) $(8,9)$. Extensive neointimal hyperplasia composed of VSMCs has been observed at the anastomosis site of AVF (10). Thus, inhibiting the viability and migration of VSMCs may be an effective intervention of AVF stenosis. It has been confirmed that activation of the PI3K/Akt signaling pathway promotes aberrant viability and migration of VSMCs $(11,12)$. Park et al $(13)$ demonstrated that inhibiting the PI3K/Akt signaling pathway disrupts the viability of rat aortic VSMCs. Conversely, activation of the PI3K/Akt signaling pathway induces the viability and migration of VSMCs (14). Therefore, therapies targeting the PI3K/Akt signaling pathway may be promising in inhibiting AVF stenosis.

Hydroxysafflor Yellow A (HSYA) is a water-soluble chalcone glycoside extracted from Carthami Flos, the flower of safflower (Carthamus tinctorius L.), which is the primary active ingredient in the pharmacological action of Carthami Flos (15). HSYA exerts several pharmacological effects, such as cardiovascular effects (16), neuroprotective effects (17), 
antitumor effects (18) and endothelium cell protection (19). Jiang et al (20) reported that HSYA suppresses the viability, migration and invasion of lipopolysaccharide-induced non-small cell lung cancer cells by suppressing the PI3K/AKT/mTOR signaling pathway. Previous studies have demonstrated that HSYA inhibits the viability and migration of VSMCs by regulating Akt signaling $(21,22)$. However, whether HSYA can modulate AVF stenosis in patients with CRF via inhibiting VSMC viability and migration remains largely unknown.

In the present study, VSMCs were induced using serum from CRF rats, and the aim was to assess the effects of HSYA on the viability and migration of VSMCs, as well as uncover the potential mechanisms.

\section{Materials and methods}

Animal model of CRF. Animal experiments were performed as previously described (23). A total of 40 male Wistar rats (age, 6-7 weeks; weight, 160-180 g) were obtained from Nanjing Jiancheng Bioengineering Institute, and maintained in a $12 \mathrm{~h}$ light/dark cycle, with $50-60 \%$ humidity at $22-26^{\circ} \mathrm{C}$. All rats were provided ad libitum access to a standard diet and water. Rats were randomly divided into two groups; a control $(n=20)$ and CRF $(n=20)$ groups after 1 week of adaptive feeding. Adenine (2.5 g; Sigma-Aldrich; Merck KGaA) was added to $100 \mathrm{ml}$ normal saline to prepare a $2.5 \%$ adenine suspension. Rats in the CRF group received $250 \mathrm{mg} / \mathrm{kg}$ adenine once a day via oral gavage for a total of 14 days, and adenine was administrated every other day for the next 14 days. Rats in the control group received the same amount of normal saline. All rats were fasted for $12 \mathrm{~h}$ prior to the last administration. Rats were anesthetized with $2 \%$ sodium pentobarbital $(50 \mathrm{mg} / \mathrm{kg})$ $1 \mathrm{~h}$ after the final administration, and cervical dislocation of the spine was immediately performed following collection of 4-6 $\mathrm{ml}$ blood from the abdominal aorta. All animal experiments were approved by the Experimental Animal Center of Lianyungang Hospital of Traditional Chinese Medicine (Lianyungang, China; approval. no. IACUC-20200312-07).

Serum parameters. All experiments were performed as previously described (23-26). Blood samples were centrifuged at $1,000 \mathrm{x} \mathrm{g}$ for $10 \mathrm{~min}$ to collect serum. The concentrations of serum creatinine ( $\mathrm{SCr}$ ) and blood urea nitrogen (BUN) were measured using commercial kits (cat. nos. C011-2-1 and C013-2-1, respectively; Nanjing Jiancheng Bioengineering Institute), and measured using a biochemical autoanalyzer (ROCHE Modular P800; Roche Diagnostics GmbH).

Cell culture and treatment. Human umbilical vein smooth muscle cells (HUVSMCs; cat. no. CP-H084) were purchased from Procell Life Science \& Technology Co., Ltd., with the approval of Ethics Committee of Lianyungang Hospital of Traditional Chinese Medicine (Lianyungang, China. Approval no. IACUC-20200611-03), and maintained in DMEM supplemented with 10\% FBS (both purchased from Gibco; Thermo Fisher Scientific, Inc.), at $37^{\circ} \mathrm{C}$ with $5 \% \mathrm{CO}_{2}$.

The rat whole blood was collected from the control (control serum) and CRF (CRF serum) groups into coagulation tubes and allowed to clot on ice for $50 \mathrm{~min}$. Subsequently, centrifugation at $1,000 \mathrm{x}$ for $15 \mathrm{~min}$ was used for separating serum. Rat serum was maintained in DMEM at $37^{\circ} \mathrm{C}$ for $48 \mathrm{~h}$ at dilutions of 2.5:100, 5:100 or 10:100. HUVSMCs were pretreated with 1,5 or $25 \mu \mathrm{M}$ HSYA (27-30) (purity $>98 \%$; Beijing Solarbio Science \& Technology Co., Ltd.) prior to stimulation of rat serum. AMG 511 (5 nM; MedChemExpress) was used to inhibit PI3K.

Cell viability assay. A Cell Counting Kit-8 (CCK-8) assay was performed to assess the effect of rat serum and/or HSYA on cell viability. HUVSMCs were seeded in 96-well plates at a density of $5 \times 10^{3}$ cells/well and treated with rat serum and/or HSYA at $37^{\circ} \mathrm{C}$ for $48 \mathrm{~h}$. Cells were subsequently incubated with $10 \mu \mathrm{l} \mathrm{CCK}-8$ reagent (cat. no. C0037; Beyotime Institute of Biotechnology) for $1 \mathrm{~h}$ at $37^{\circ} \mathrm{C}$. Cell viability was analyzed at a wavelength of $450 \mathrm{~nm}$, using a microplate spectrophotometer (BioTek Instruments, Inc.).

Wound healing assay. HUVSMCs were seeded into 6-well plates at a density of $1 \times 10^{6}$ cells/well. Sterile $200 \mu 1$ pipette tips were used to scratch the cell monolayers. Cell medium was replaced with fresh serum-free DMEM. The migratory ability of cells characterized by wound width was observed at 0 and $48 \mathrm{~h}$ under a light microscope (Olympus Corporation; magnification, x100).

Apoptosis analysis. HUVSMCs $\left(5 \times 10^{5}\right)$ were collected by centrifugation at $37^{\circ} \mathrm{C}$ and $300 \mathrm{x}$ g for $3 \mathrm{~min}$ and re-suspended in $200 \mu \mathrm{l}$ Annexin $\mathrm{V}$ binding buffer (cat. no. C1062M; Beyotime Institute of Biotechnology). After resuspension, cells were incubated with $10 \mu \mathrm{l}$ PI (cat. no. C1062M; Beyotime Institute of Biotechnology) for $15 \mathrm{~min}$ at room temperature in the dark. Apoptotic cells were subsequently analyzed using a flow cytometer (Beckman Coulter, Inc.).

Measurement of nitric oxide (NO). HUVSMCs were seeded in 12 -well plates at a density of $2 \times 10^{5}$ cells/well and treated with rat serum and/or HSYA. HUVSMCs were subsequently lysed using Lysis Buffer (cat. no. S3090; Beyotime Institute of Biotechnology). The Nitrate/Nitrite assay kit (cat. no. S0023; Beyotime Institute of Biotechnology) was used to determine NO concentration in HUVSMCs, according to the manufacturer's protocol. NO concentration was measured at a wavelength of $540 \mathrm{~nm}$, using a microplate spectrophotometer (BioTek Instruments, Inc.).

Western blotting. Total protein was extracted from HUVSMCs using RIPA lysis buffer (cat. no. P0013B; Beyotime Institute of Biotechnology). Cell supernatants were collected by centrifugation at $12,000 \mathrm{x} \mathrm{g}$ for $15 \mathrm{~min}$ at $4^{\circ} \mathrm{C}$. Total protein was quantified using the BCA protein assay kit (cat. no. P0012s; Beyotime Institute of Biotechnology) and $20 \mu \mathrm{g}$ protein/lane was separated by $12 \%$ SDS-PAGE. The separated proteins were subsequently transferred to PVDF membranes (EMD Millipore) and blocked with 5\% skimmed milk for $2 \mathrm{~h}$ at room temperature. The membranes were incubated with primary antibodies against PI3K (1:1,000), Akt (1:1,000), phosphorylated (p)-Akt (cat. no. Ser473, 1:1,000), endothelial NO synthase (eNOS; 1:1,000), p-eNOS (1:1,000) and GAPDH $(1: 10,000)$ overnight at $4^{\circ} \mathrm{C}$ (all purchased from Affinity Biosciences). 
A

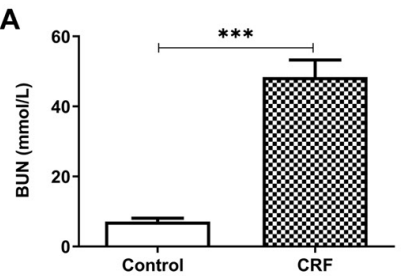

B

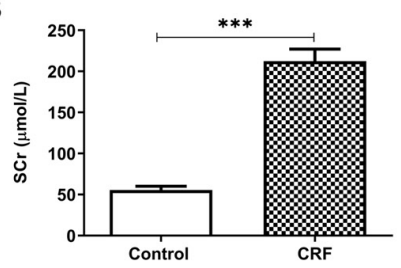

C

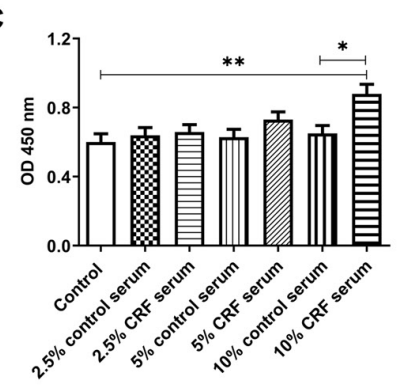

E

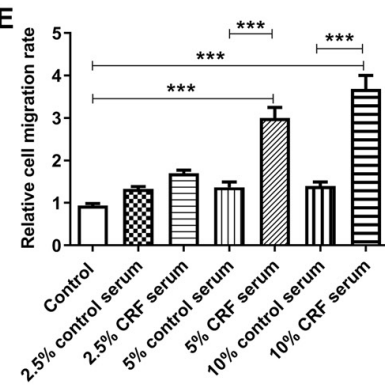

D

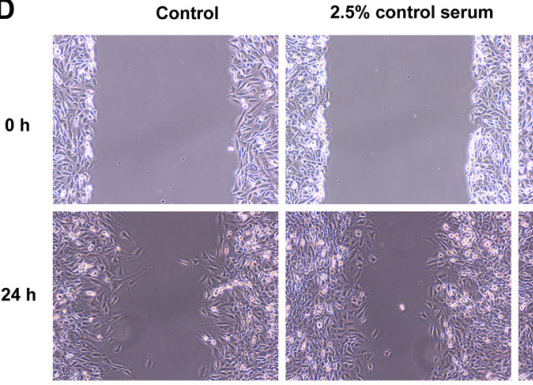

$2.5 \%$ CRF serum

$5 \%$ control serum

$5 \%$ CRF serum

$10 \%$ control serum

$10 \%$ CRF serum
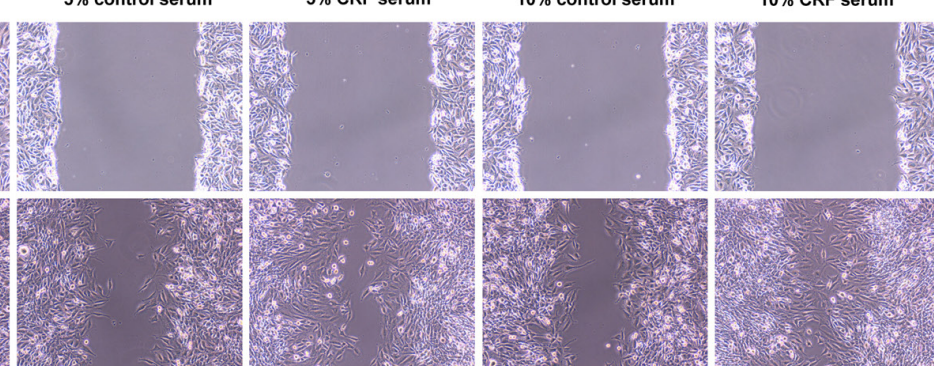

Figure 1. Serum from CRF rats promotes viability and migration of HUVSMCs. (A and B) Concentrations of BUN and SCr in the rat serum of the control or CRF group. (C) HUVSMCs were stimulated with different concentrations of serum from the control or CRF group. The viability of HUVSMCs was evaluated via examining the absorbance at $450 \mathrm{~nm}$. (D and E) Migratory capability of HUVSMCs was determined using a wound healing assay and the relative migratory rate was quantified. ${ }^{*} \mathrm{P}<0.05,{ }^{* *} \mathrm{P}<0.01$ and ${ }^{* * * *} \mathrm{P}<0.001$. SCr, serum creatinine; BUN, blood urea nitrogen; $\mathrm{CRF}$, chronic renal failure; HUVSMCs, human umbilical vein smooth muscle cells; OD, optical density.

Following the primary incubation, membranes were incubated with goat anti-rabbit/mouse IgG $(\mathrm{H}+\mathrm{L})$ HRP-conjugated secondary antibodies (1:10,000; Affinity Biosciences) for $2 \mathrm{~h}$ at room temperature. Protein bands were semi-quantified using Image Lab version 4.1 software (Bio-Rad Laboratories, Inc.).

Statistical analysis. All experiments were performed in triplicate and data are presented as the mean \pm standard deviation. A Student's t-test was used to compare differences between two groups, and a one-way ANOVA followed by a Tukey's post hoc test was used to compare differences between multiple groups in GraphPad Prism version 5.0 (GraphPad Software, Inc.). $\mathrm{P}<0.05$ was considered to indicate a statistically significant difference.

\section{Results}

Serum from CRF rats promotes the viability and migration of HUVSMCs. Rat serum from the control and CRF groups was collected to assess changes in the parameters of renal function. As presented in Fig. 1A, the BUN concentration was significantly higher in the CRF group compared with the control group. Similarly, SCr concentration was higher in the CRF group compared with the control group (Fig. 1B). These results suggest that the renal failure model was established successfully (24-26).

HUVSMCs were treated with rat serum to assess the effect of CRF serum on HUVSMCs. The results demonstrated that $10 \%$ CRF serum significantly promoted cell viability compared with $10 \%$ control serum, whereas low concentrations of CRF serum (2.5 and 5.0\%) slightly enhanced cell viability (Fig. 1C). In addition, the migratory ability of HUVSMCs was inhibited following stimulation of CRF serum compared with the same concentration of control serum. Furthermore, the results of the wound healing assay demonstrated that CRF serum promoted cell migration in a concentration-dependent manner, whereas control serum had little effect on cell migration (Fig. 1D and E). Collectively, these results suggest that serum from CRF rats promotes the viability and migration of HUVSMCs.

Serum from CRF rats promotes the apoptosis of HUVSMCs. The results demonstrated that the apoptotic rate increased following treatment of HUVSMCs with rat serum. Notably, $10 \%$ CRF serum markedly promoted cell apoptosis compared with $10 \%$ control serum (Fig. 2A). This may be explained by the presence of toxic substances in the serum of rats with CRF, which affects cell survival and promotes apoptosis.

HSYA inhibits cell viability, migration and apoptosis of HUVSMCs. HUVSMCs were treated with HSYA or AMG 511 in the presence of $10 \%$ CRF serum. As presented in Fig. 3A, HSYA inhibited cell viability induced by $10 \%$ CRF serum in a concentration-dependent manner. Similarly, AMG 511 suppressed the viability of HUVSMCs. Notably, pretreatment with HSYA or AMG 511 alleviated 10\% CRF serum-induced cell migration (Fig. 3B), suggesting that HSYA reverses the effect of CRF serum on cell migration. In addition, HSYA partially alleviated $10 \% \mathrm{CRF}$ serum-induced apoptosis, the effects of which were reversed following pretreatment with AMG 511 (Fig. 3C). Taken together, these results suggest that HSYA counteracts the effects of CRF serum on the viability, migration and apoptosis of HUVSMCs.

HSYA inactivates the PI3K/Akt signaling pathway and promotes $N O$ production. NO inhibits neointimal hyperplasia (31); thus, the NO concentration in HUVSMCs was detected. The NO concentration was notably decreased in 
A

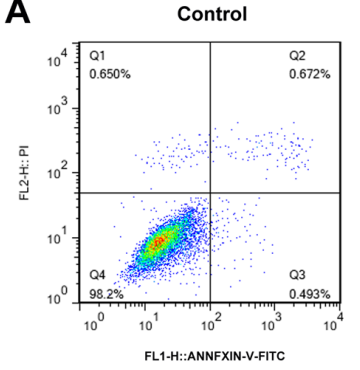

$5 \%$ CRF serum

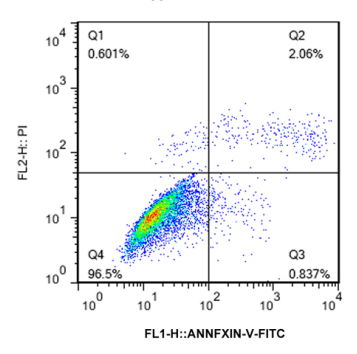

$2.5 \%$ control serum

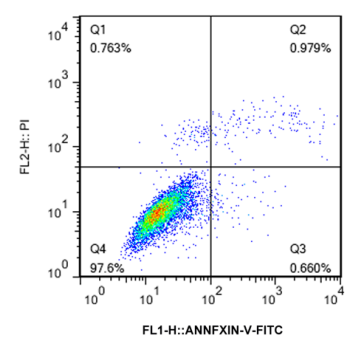

$10 \%$ control serum

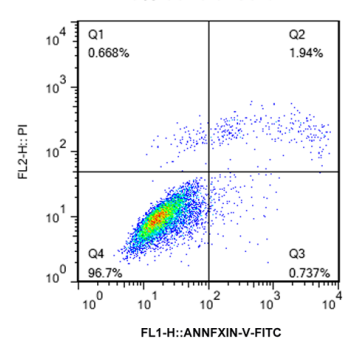

$2.5 \%$ CRF serum

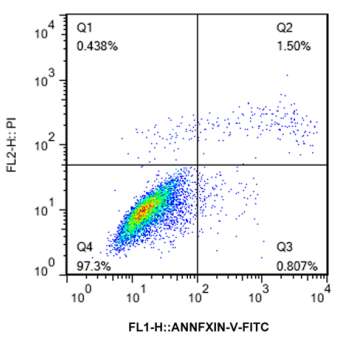

$10 \%$ CRF serum

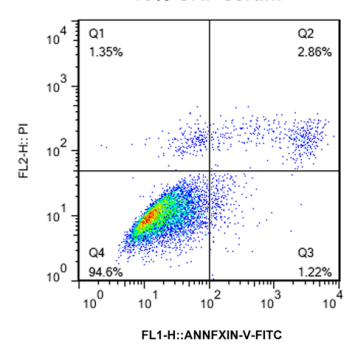

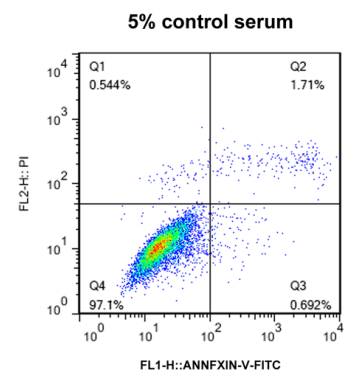

B

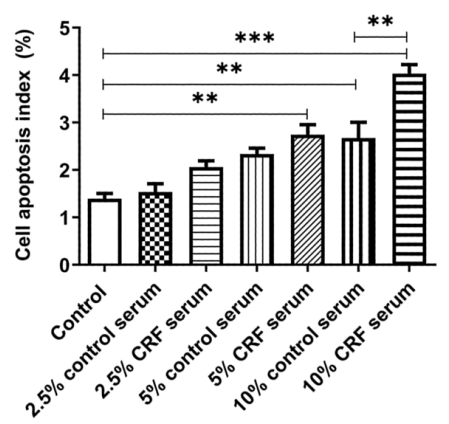

Figure 2. Serum from CRF rats enhances apoptosis of HUVSMCs. HUVSMCs were stimulated with 2.5, 5 or $10 \%$ serum from the control or CRF group. (A) Apoptotic ratio of each group was detected via flow cytometry. (B) Quantitative analysis of flow cytometry. ${ }^{* *} \mathrm{P}<0.01$ and ${ }^{* * *} \mathrm{P}<0.001$. CRF, chronic renal failure; HUVSMCs, human umbilical vein smooth muscle cells.

the presence of $10 \%$ CRF serum compared with the control group; however, treatment with HSYA elevated NO levels in a concentration-dependent manner. Similarly, treatment with AMG 511 promoted NO production (Fig. 4A).

Given that HSYA exhibited similar inhibitory effects to AMG 511 on the viability and migration of HUVSMCs, it was next investigated whether HSYA can exert these effects by inhibiting the PI3K/Akt pathway. As presented in Fig. 4B, 10\% CRF serum upregulated PI3K expression and phosphorylation of Akt, suggesting that serum from CRF rats can activate PI3K/Akt signaling in HUVSMCs. Notably, the expression levels of PI3K and p-Akt decreased following treatment with AMG 511. In addition, treatment with HSYA markedly decreased the protein expression levels of PI3K and p-Akt. Treatment with both HSYA and AMG 511 upregulated p-eNOS expression, respectively, which explains the increase in NO production (Fig. 4A).

\section{Discussion}

AVF stenosis caused by neointimal hyperplasia is frequently observed in patients (32), and can lead to the morbidity of patients with ESRD $(33,34)$. However, the molecular mechanism underlying neointima formation in AVF remains unclear. It is well-known that injury induced pathological viability and migration of VSMCs is a major cause of neointima formation $(35,36)$. In the present study, CRF rats were established and the serum from rats were prepared to stimulate HUVSMCs to determine whether HSYA could inhibit neointimal hyperplasia in vitro.

High concentrations of CRF serum significantly promoted the viability and migration of HUVSMCs, which is consistent with the pathological condition of AVF stenosis in patients with ESRD $(37,38)$. However, high concentrations of CRF serum also increase cell apoptosis, which may be due to toxicants in the serum that disrupt cell survival. Activation of the PI3K/Akt signaling pathway is closely associated with aberrant viability and migration of VSMCs $(11,12,39)$. In addition, activation of the PI3K/Akt signaling pathway has been observed in mice with renal dysfunction caused by ischemia/reperfusion-injury $(40,41)$. Previous studies have reported that the PI3K/Akt signaling pathway is activated in kidney injury induced by cisplatin (42) and kidneys of rats with unilateral ureteral obstruction (43).

HSYA is an active ingredient isolated from Carthami Flos (15). It has been reported that HSYA inhibits platelet derived growth factor BB-induced activation of Akt signaling, which in-turn disrupts the viability and migration of VSMCs (21). In addition, Yang et al (22) demonstrated that HSYA suppresses the viability and migration of lipopolysaccharide-induced VSMCs by inhibiting the Toll-like receptor 4/Rac1/Akt pathway. Thus, it was hypothesized that HSYA exerts antiproliferative and anti-migratory effects on HUVSMCs via the PI3K/Akt signaling pathway.

The results of the present study demonstrated that HSYA suppressed CRF serum-induced cell viability and migration, suggesting its role in HUVSMCs-mediated intimal hyperplasia. AMG 511, which is a potent and selective PI3K inhibitor that decreases the phosphorylation of Akt, exhibited similar effects to HSYA on the viability and migration of HUVSMCs. Notably, HSYA decreased cell apoptosis induced by CRF serum, whereas AMG 511 had little effect on the apoptosis of HUVSMCs. This may be explained by the hypothesis that HSYA ameliorates the toxic effects of toxicants in CRF serum on HUVSMCs.

In the present study, NO production and p-eNOS expression decreased in CRF serum-induced HUVSMCs, whereas treatment with HSYA and AMG 511 enhanced the levels of 
A

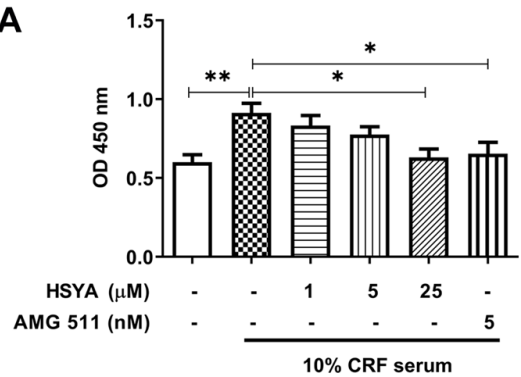

C

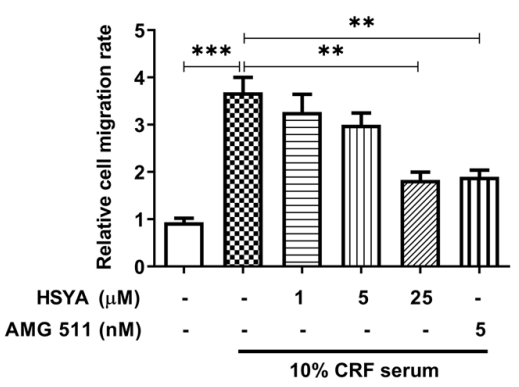

B

B

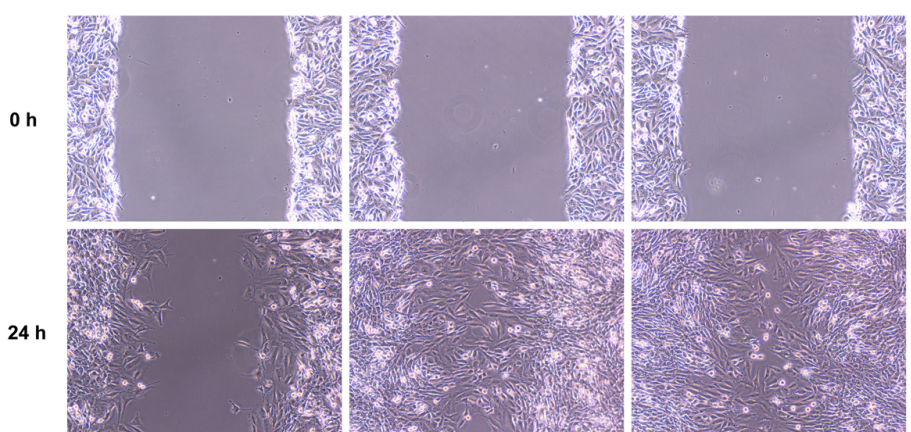

HSYA $5(\mu \mathrm{M})$
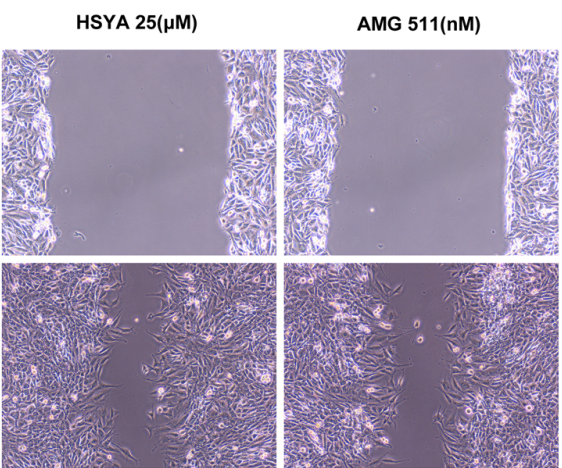

D
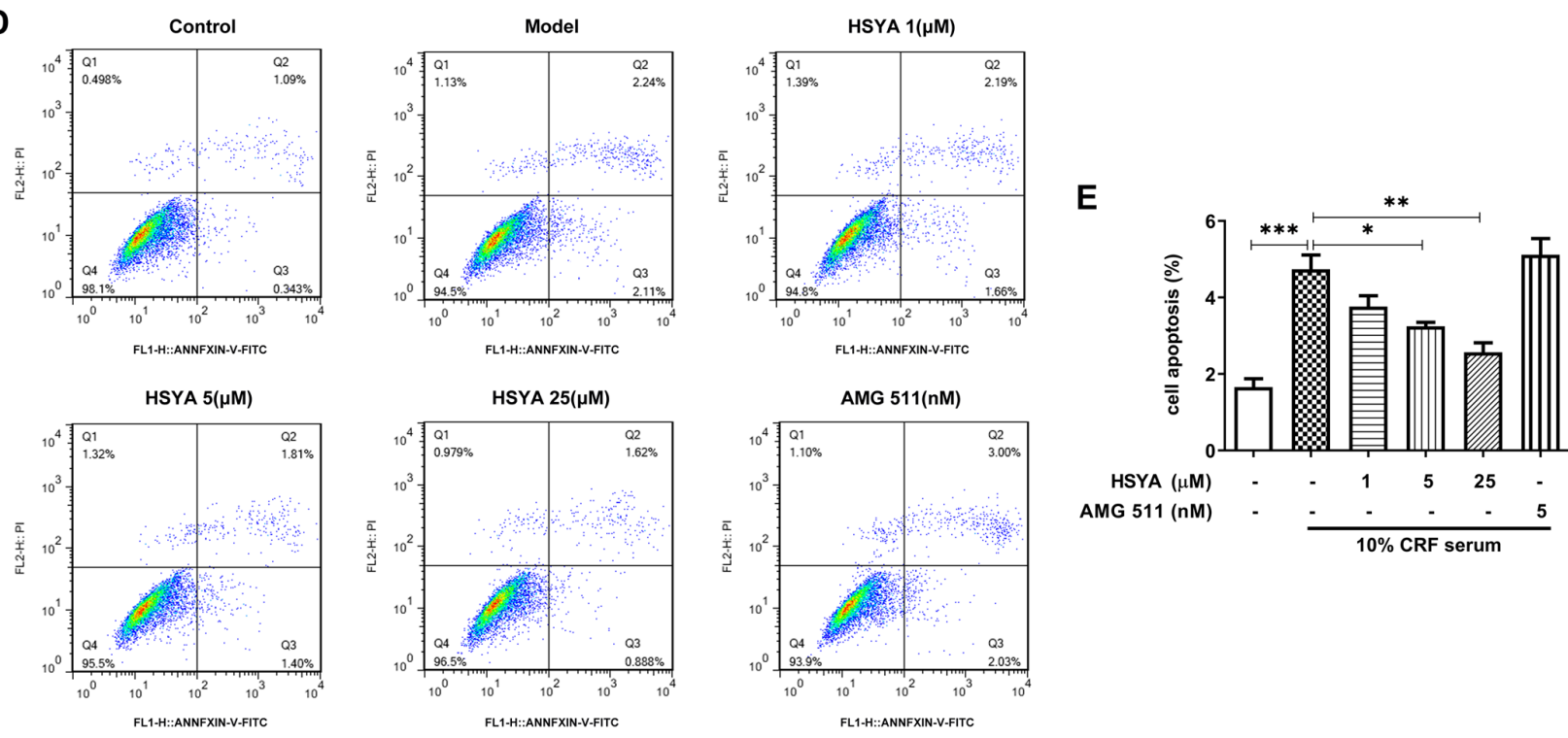

Figure 3. HSYA inhibits cell viability, migration and apoptosis. Solutions of 1, 5, $25 \mu$ M HSYA or 5 nM AMG 511 were prepared to treat HUVSMCs 30 min prior to stimulation with $10 \%$ CRF serum. (A) Viability of HUVSMCs was evaluated via examining the absorbance at 450 nm. (B and C) Migratory capability of HUVSMCs was determined using a wound healing assay and the relative migratory rate was quantified. (D and E) Apoptotic ratio of each group was detected via flow cytometry. ${ }^{*} \mathrm{P}<0.05,{ }^{* *} \mathrm{P}<0.01$ and ${ }^{* * * *} \mathrm{P}<0.001$. HSYA, Hydroxysafflor yellow A; CRF, chronic renal failure; HUVSMCs, human umbilical vein smooth muscle cells; OD, optical density.

NO and p-eNOS, respectively. Previous studies have demonstrated that NO-based therapies can decrease neointimal hyperplasia $(44,45)$. NO production is regulated by NO synthases (NOSs), including eNOS. eNOS activity is mainly regulated through phosphorylation, which is primarily regulated by the PI3K/Akt/eNOS pathway. It has been reported that activation of PI3K/AKT/eNOS pathways serves an important role in regulating cell migration, migration, angiogenesis and apoptosis (46). The results of the present study showed that HSYA may rescue NO production in CRF serum-treated cells via inhibiting PI3K/Akt activation.

To further investigate whether HSYA can regulate the PI3K/Akt signaling pathway, the protein expression levels of
PI3K and p-Akt were detected. The results demonstrated that HSYA affected PI3K expression and Akt phosphorylation. In addition, HSYA inhibited the viability and migration of HUVSMCs by regulating PI3K/Akt signaling, which suggests that HSYA may mediate intimal hyperplasia-induced AVF stenosis. However, given that the present study only focused on HSYA-mediated viability and migration of HUVSMCs, further studies are required to confirm the effects of HSYA in AVF stenosis in vivo. In addition, other alternative assays will be utilized to enrich the experimental content and further validate these findings. Finally, prospective studies should focus on investigating the involvement of other pathways on the effects of HSYA on AVF stenosis. 

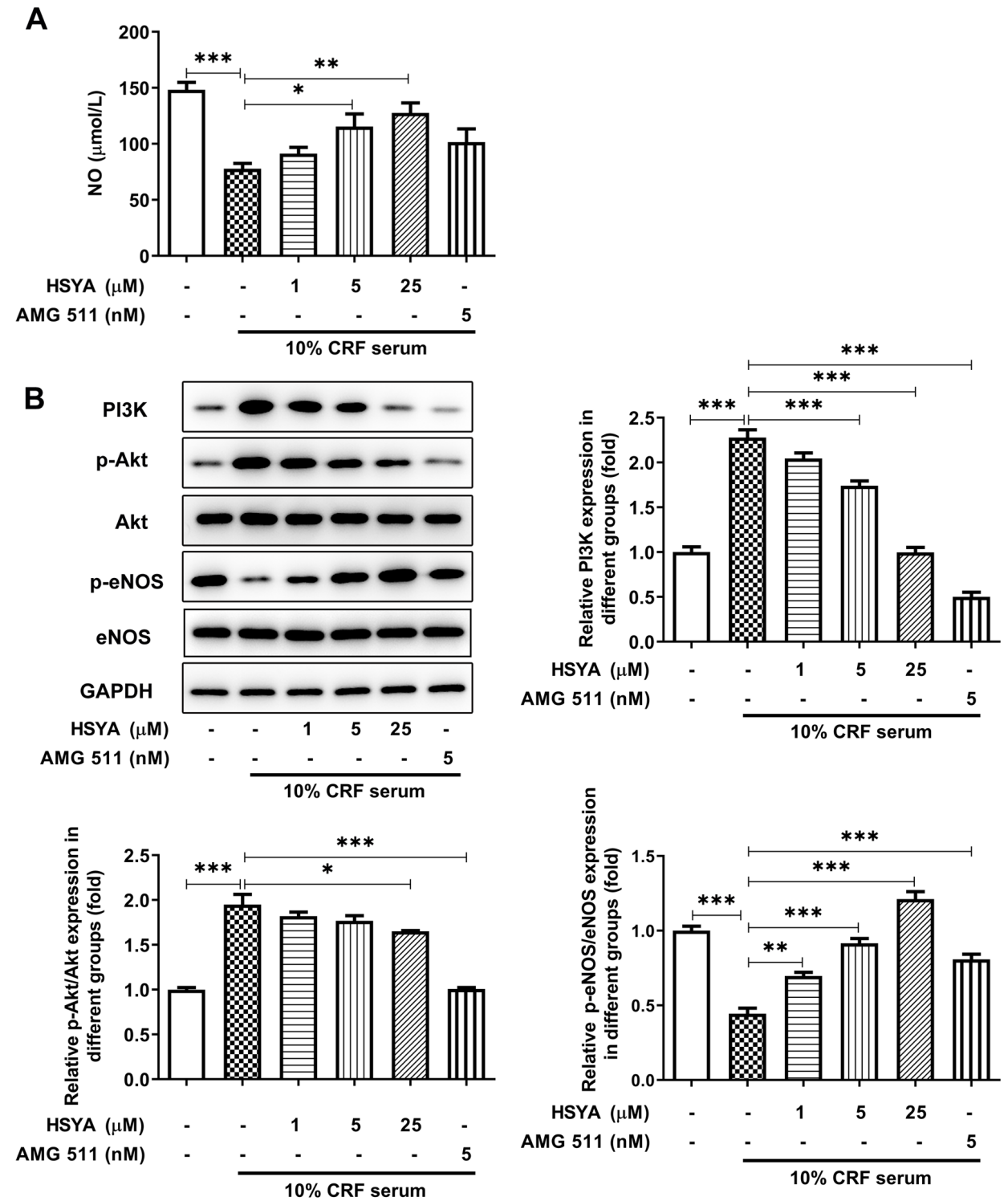

Figure 4. HSYA inactivates PI3K/Akt signaling and enhances NO production. Solutions of 1, 5, 25 $\mu \mathrm{M}$ HSYA or 5 nM AMG 511 were prepared to treat HUVSMCs 30 min prior to stimulation with $10 \%$ CRF serum. (A) Production of NO in each group was examined using a Nitrate/Nitrite assay kit. (B) Protein levels of PI3K, Akt, p-Akt, eNOS and p-eNOS were estimated via western blotting. ${ }^{*} \mathrm{P}<0.05,{ }^{* * *} \mathrm{P}<0.01$ and ${ }^{* * *} \mathrm{P}<0.001$. HSYA, Hydroxysafflor yellow A; CRF, chronic renal failure; HUVSMCs, human umbilical vein smooth muscle cells; p-, phospho-; NO, nitric oxide; eNOS, endothelial NO synthase.

Taken together, the current study for the first time demonstrated the inhibitory effects of HSYA on HUVSMC viability and migration, as well as showing the underlying mechanism involved regulation of the PI3K/Akt signaling pathway. The results provide primary evidence for the therapeutic application of HSYA in intimal hyperplasia-induced AVF stenosis.

\section{Acknowledgements}

Not applicable.

\section{Funding}

The present study was supported by the Six One Project of Top-notch Talent item for High-level Health Talents in Jiangsu Province in 2017 (grant. no. LGY 2017064) and the Fifth Phase of the 333 Project for Scientific Research Project of Jiangsu Province in 2020 (grant. no. BRA2020259).

\section{Availability of data and materials}

All data generated or analysed during the present study are included in this published article.

\section{Authors' contributions}

$\mathrm{BC}, \mathrm{CH}, \mathrm{LW}$ and $\mathrm{QW}$ conceived and designed the present study. $\mathrm{CH}$ and $\mathrm{LW}$, performed the experiments and acquired the data. QW and YY analyzed the data. BC drafted the initial manuscript, including the figures. All authors have read and approved the final manuscript. $\mathrm{BC}$ and $\mathrm{CH}$ confirm the authenticity of all the raw data.

\section{Ethics approval and consent to participate}

All animal experiments were approved by the Experimental Animal Center of Lianyungang Hospital of Traditional Chinese 
Medicine (Lianyungang, China. Approval. no. IACUC-20200 312-07). The use of Human umbilical vein smooth muscle cells (cat. no. CP-H084; Procell Life Science \& Technology Co., Ltd.) was approved by the Ethics Committee of Lianyungang Hospital of Traditional Chinese Medicine (Lianyungang, China. Approval. no. IACUC-20200611-03).

\section{Patient consent for publication}

Not applicable.

\section{Competing interests}

The authors declare that they have no competing interests.

\section{References}

1. Mallick NP and Gokal R: Haemodialysis. Lancet (London, England) 353: 737-742, 1999

2. Radosa CG, Radosa JC, Weiss N, Schmidt C, Werth S, Hofmockel T, Plodeck V, Gatzweiler C, Laniado M and Hoffmann RT: Endovascular creation of an arteriovenous fistula (endoAVF) for hemodialysis access: First results. Cardiovasc Intervent Radiol 40: 1545-1551, 2017.

3. Gh K, Mhs M, Ravari H, Daliri M, Hoseini L and Nateghi M: Primary patency rate of native AV fistula: Long term follow up. Int J Clin Exp Med 5: 173-178, 2012.

4. Dixon BS: Why don't fistulas mature? Kidney Int 70: 1413-1422, 2006.

5. Sullivan KL, Besarab A, Bonn J, Shapiro MJ, Gardiner GA Jr and Moritz MJ: Hemodynamics of failing dialysis grafts. Radiology 186: 867-872, 1993.

6. Bashar K, Conlon PJ, Kheirelseid EA, Aherne T, Walsh SR and Leahy A: Arteriovenous fistula in dialysis patients: Factors implicated in early and late AVF maturation failure. Surgeon 14: 294-300, 2016.

7. Langer S, Kokozidou M, Heiss C, Kranz J, Kessler T, Paulus N, Krüger T, Jacobs MJ, Lente C and Koeppel TA: Chronic kidney disease aggravates arteriovenous fistula damage in rats. Kidney Int 78: 1312-1321, 2010

8. Low EL, Baker AH and Bradshaw AC: TGF $\beta$, smooth muscle cells and coronary artery disease: A review. Cell Signal 53: 90-101, 2019

9. Yue Y, Ma K, Li Z and Wang Z: Angiotensin II type 1 receptor-associated protein regulates carotid intimal hyperplasia through controlling apoptosis of vascular smooth muscle cells. Biochem Biophys Res Commun 495: 2030-2037, 2018.

10. Zhao J, Jourd'heuil FL, Xue M, Conti D, Lopez-Soler RI, Ginnan R, Asif A, Singer HA, Jourd'heuil D and Long X: Dual function for mature vascular smooth muscle cells during arteriovenous fistula remodeling. J Am Heart Assoc 30: e004891, 2017.

11. Liu C, Su T, Li F, Li L, Qin X, Pan W, Feng F, Chen F, Liao D and Chen L: PI3K/Akt signaling transduction pathway is involved in rat vascular smooth muscle cell viability induced by apelin-13 Acta Biochim Biophys Sin (Shanghai) 42: 396-402, 2010.

12. Gerthoffer WT: Mechanisms of vascular smooth muscle cell migration. Circ Res 100: 607-621, 2007.

13. Park ES, Kang SI, Yoo KD, Lee MY, Yoo HS, Hong JT, Shin HS, Kim B and Yun YP: Camptothecin inhibits platelet-derived growth factor-BB-induced viability of rat aortic vascular smooth muscle cells through inhibition of PI3K/Akt signaling pathway. Exp Cell Res 319: 982-991, 2013.

14. Fang H, Yang S, Luo Y, Zhang C, Rao Y, Liu R, Feng Y and Yu J: Notoginsenoside R1 inhibits vascular smooth muscle cell viability, migration and neointimal hyperplasia through PI3K/Akt signaling. Sci Rep 8: 7595, 2018.

15. Ao H, Feng W and Peng C: Hydroxysafflor yellow A: A promising therapeutic agent for a broad spectrum of diseases. Evid Based Complement Alternat Med 2018: 8259280, 2018.

16. Bai Y, Lu P, Han C, Yu C, Chen M, He F, Yi D and Wu L: Hydroxysafflor yellow A (HSYA) from flowers of carthamus tinctorius L. and its vasodilatation effects on pulmonary artery. Molecules 17: 14918-14927, 2012.
17. Chen L, Xiang Y, Kong L, Zhang X, Sun B, Wei X and Liu H: Hydroxysafflor yellow A protects against cerebral ischemia-reperfusion injury by anti-apoptotic effect through PI3K/Akt/GSK3 $\beta$ pathway in rat. Neurochem Res 38: 2268-2275, 2013.

18. Xiao J, Lv Y, Jin F, Liu Y, Ma Y, Xiong Y, Liu L, Zhang S, Sun Y, Tipoe GL, et al: LncRNA HANR promotes tumorigenesis and increase of chemoresistance in hepatocellular carcinoma. Cell Physiol Biochem 43: 1926-1938, 2017.

19. Ye F, Wang J, Meng W, Qian J and Jin M: Proteomic investigation of effects of hydroxysafflor yellow A in oxidized low-density lipoprotein-induced endothelial injury. Sci Rep 7: 17981, 2017.

20. Jiang M, Zhou LY, Xu N and An Q: Hydroxysafflor yellow A inhibited lipopolysaccharide-induced non-small cell lung cancer cell proliferation, migration, and invasion by suppressing the $\mathrm{PI} 3 \mathrm{~K} / \mathrm{AKT} / \mathrm{mTOR}$ and ERK/MAPK signaling pathways. Thorac Cancer 10: 1319-1333, 2019.

21. Song Y, Long L, Zhang N and Liu Y: Inhibitory effects of hydroxysafflor yellow A on PDGF-BB-induced viability and migration of vascular smooth muscle cells via mediating akt signaling. Mol Med Rep 10: 1555-1560, 2014.

22. Yang G, Zhou X, Chen T, Deng Y, Yu D, Pan S and Song Y: Hydroxysafflor yellow A inhibits lipopolysaccharide-induced viability and migration of vascular smooth muscle cells via toll-like receptor-4 pathway. Int J Clin Exp Med 8: 5295-5302, 2015

23. Chen J, Shi W, Xu Y, Zhang H and Chen B: Hirudin prevents vascular endothelial cell apoptosis and permeability enhancement induced by the serum from rat with chronic renal failure through inhibiting RhoA/ROCK signaling pathway. Drug Dev Res 20: doi:10.1002, 2020

24. Zhang G, Cui G, Tong S and Cao Q: Salvianolic acid A alleviates the renal damage in rats with chronic renal failure1. Acta Cir Bras 34: e201900204, 2019.

25. Xue L, Pan Z, Yin Q, Zhang P, Zhang J and Qi W: Liraglutide promotes autophagy by regulating the AMPK/mTOR pathway in a rat remnant kidney model of chronic renal failure. Int Urol Nephrol 51: 2305-2313, 2019.

26. Ngai HH, Sit WH and Wan JM: The nephroprotective effects of the herbal medicine preparation, $\mathrm{WH} 30+$, on the chemical-induced acute and chronic renal failure in rats. Am J Chin Med 33: 491-500, 2005.

27. Chen S, Ma J, Zhu H, Deng S, Gu M and Qu S: Hydroxysafflor yellow A attenuates high glucose-induced human umbilical vein endothelial cell dysfunction. Hum Exp Toxicol 38: 685-693, 2019.

28. Sun L, Xu YW, Han J, Xiao C, Cao SS, Liang H and Cheng Y: Hydroxysafflor yellow A shows protection against PPAR $\gamma$ inactivation in nitrosative neurons. Oxid Med Cell Longev 2018: 9101740, 2018.

29. Chen Z, Liu L, Liu Y, Wang S, Zhang S, Dong R, Xu M, Ma Y, Wang J, Zhang Q and Wei P: Hydroxysafflor yellow A induces autophagy in human liver cancer cells by regulating beclin 1 and ERK expression. Exp Ther Med 19: 2989-2996, 2020.

30. Guo X, Zheng M, Pan R, Zang B, Gao J, Ma H and Jin M: Hydroxysafflor yellow A (HSYA) targets the platelet-activating factor (PAF) receptor and inhibits human bronchial smooth muscle activation induced by PAF. Food Funct 10: 4661-4673, 2019.

31. Bahnson ES, Koo N, Cantu-Medellin N, Tsui AY, Havelka GE, Vercammen JM, Jiang Q, Kelley EE and Kibbe MR: Nitric oxide inhibits neointimal hyperplasia following vascular injury via differential, cell-specific modulation of SOD-1 in the arterial wall. Nitric Oxide 44: 8-17, 2015

32. Rothuizen TC, Wong C, Quax PH, van Zonneveld AJ, Rabelink TJ and Rotmans JI: Arteriovenous access failure: More than just intimal hyperplasia? Nephrol Dial Transplant 28: 1085-1092, 2013.

33. Rotmans JI, Pasterkamp G, Verhagen HJ, Pattynama PM, Blankestijn PJ and Stroes ES: Hemodialysis access graft failure: Time to revisit an unmet clinical need? J Nephrol 18: 9-20, 2005.

34. Kokubo $T$, Ishikawa $N$, Uchida $H$, Chasnoff SE, Xie X, Mathew S, Hruska KA and Choi ET: CKD accelerates development of neointimal hyperplasia in arteriovenous fistulas. J Am Soc Nephrol 20: 1236-1245, 2009.

35. Marx SO, Totary-Jain H and Marks AR: Vascular smooth muscle cell viability in restenosis. Circ Cardiovasc Interv 4: 104-111, 2011.

36. Rzucidlo EM, Martin KA and Powell RJ: Regulation of vascular smooth muscle cell differentiation. J Vas Surg 45 (Suppl A): A25-A32, 2007.

37. Dember LM, Beck GJ, Allon M, Delmez JA, Dixon BS, Greenberg A, Himmelfarb J, Vazquez MA, Gassman JJ, Greene T, et al: Effect of clopidogrel on early failure of arteriovenous fistulas for hemodialysis: A randomized controlled trial. JAMA 299: 2164-2171, 2008 
38. Al-Jaishi AA, Oliver MJ, Thomas SM, Lok CE, Zhang JC, Garg AX, Kosa SD, Quinn RR and Moist LM: Patency rates of the arteriovenous fistula for hemodialysis: A systematic review and meta-analysis. Am J Kidney Dis 63: 464-478, 2014.

39. Tang F, Liu M, Zeng O, Tan W, Long J, Liu S, Yang J and Chu C: Gefitinib-coated balloon inhibits the excessive hyperplasia of intima after vascular injuries through PI3K/AKT pathway. Technol Health Care 27: 331-343, 2019.

40. Zhu J, Chen X, Wang H and Yan Q: Catalpol protects mice against renal ischemia/reperfusion injury via suppressing PI3K/Akt-eNOS signaling and inflammation. Int J Clin Exp Med 8: 2038-2044, 2015.

41. Hu S, Zhang Y, Zhang M, Guo Y, Yang $\mathrm{P}$, Zhang S, Simsekyilmaz S, Xu JF, Li J, Xiang X, et al: Aloperine protects mice against ischemia-reperfusion (IR)-induced renal injury by regulating PI3K/AKT/mTOR signaling and AP-1 activity. Mol Med 21: 912-923, 2016.

42. Potočnjak I and Domitrović R: Carvacrol attenuates acute kidney injury induced by cisplatin through suppression of ERK and PI3K/Akt activation. Food Chem Tox 98: 251-261, 2016.
43. Ma SK, Joo SY, Kim CS, Choi JS, Bae EH, Lee J and Kim SW: Increased hosphorylation of PI3K/Akt/mTOR in the obstructed kidney of rats with unilateral ureteral obstruction. Chonnam Med J 49: 108-112, 2013.

44. Ahanchi SS, Tsihlis ND and Kibbe MR: The role of nitric oxide in the pathophysiology of intimal hyperplasia. J Vasc Surg 45 Suppl A: A64-A73, 2007.

45. Hogg ME, Varu VN, Vavra AK, Popowich DA, Banerjee MN, Martinez J, Jiang Q, Saavedra JE, Keefer LK and Kibbe MR: Effect of nitric oxide on neointimal hyperplasia based on sex and hormone status. Free Rad Biol Med 50: 1065-1074, 2011.

46. Chen R, Chen T, Wang T, Dai X, Meng K, Zhang S, Jiang D, Wang Y, Zhou K, Geng T, et al: Tongmai Yangxin pill reduces myocardial no-reflow by regulating apoptosis and activating PI3K/Akt/eNOS pathway. J Ethnopharmacol 261: 113069, 2020.

This work is licensed under a Creative Commons Attribution-NonCommercial-NoDerivatives 4.0 International (CC BY-NC-ND 4.0) License. 